\title{
Targeted Downregulation of Extracellular Nephrin in Human IgA Nephropathy
}

\author{
Elena Gagliardini ${ }^{a}$ Ariela Benigni ${ }^{a}$ Susanna Tomasoni ${ }^{a}$ Mauro Abbate ${ }^{a}$ \\ Raghu Kallurib Giuseppe Remuzzia, $c$ \\ a Mario Negri Institute for Pharmacological Research, Negri Bergamo Laboratories, Bergamo, Italy; \\ bBeth Israel Deaconess Medical Center and Harvard Medical School, Boston, Mass., USA; \\ 'Division of Nephrology and Dialysis, Azienda Ospedaliera, Ospedali Riuniti, Bergamo, Italy
}

\section{Key Words}

Nephrin - CD2-associated protein - Slit diaphragm •

Acquired glomerular disease

\begin{abstract}
Background: The identification of nephrin and CD2AP, podocyte proteins which modulate the properties of glomerular barrier selectivity, has made it possible to unravel the mechanisms undergoing foot process effacement and proteinuria. Here we explored the role of nephrin and CD2AP together with the integrity of the slit diaphragm in the pathogenesis of proteinuria in patients with acquired glomerular diseases. Methods: Nephrin mRNA and protein expression were systematically evaluated in 28 renal biopsy samples from adult patients with primary glomerular disease and proteinuria by in situ hybridization and immunohistochemistry using antibodies directed against extra- and intracellular nephrin and compared with biopsy samples from normal controls. CD2AP protein expression by immunohistochemistry was also assessed. Morphometrical analysis of the filtration slit was performed by transmission electron microscopy. Results: Nephrin mRNA and expression of the extracellular nephrin were markedly reduced in $\lg A$ nephropathy. No changes were found in patients with minimal change nephrosis and focal segmental glomerulosclerosis. The staining of intracellular nephrin and CD2AP did not change among patients. A comparable frequency of the filtration slits was observed in all patient
\end{abstract}

groups, except in minimal change disease patients, due to extensive foot process effacement. The percentage of slit diaphragms with a filamentous image was markedly reduced in IgA nephropathy, but was comparable to controls in minimal change disease. Slit pore width showed a tendency to decrease both in patients with minimal change disease and $\lg \mathrm{A}$ nephropathy. Conclusions: These results indicate that the ultrastructure of the filtration slit diaphragm is altered in patients with $\lg \mathrm{A}$ nephropathy as a consequence of targeted downregulation of extracellular nephrin. Further studies are needed to evaluate the pathophysiological meaning of nephrin abnormality in $\lg \mathrm{A}$ nephropathy and how these changes can be modulated by antiproteinuric therapy.

Copyright $\odot 2003$ S. Karger AG, Basel

\section{Introduction}

Nephrotic syndrome manifests in the most severe forms of primary and secondary glomerulopathies and often implies profound changes in glomerular podocyte morphology, including podocyte detachment from the glomerular basement membrane and loss of the interdigitating foot processes [1]. Changes in glomerular architecture and the consequent massive remodelling of the podocyte intercellular junction, the so-called slit diaphragm, alter the size-selective function of the glomerular capillary [2]. The molecular composition of the slit diaphragm was unknown for a long time until studies in familial nephrot-

\begin{tabular}{ll}
\hline KARGER & ( ) 2003 S. Karger AG, Basel \\
Fax +4161306 12 34 & \\
$\begin{array}{l}\text { E-Mail karger@karger.ch } \\
\text { www.karger.com }\end{array}$ & $\begin{array}{l}\text { Accessible online at: } \\
\text { www.karger.com/ajn }\end{array}$
\end{tabular}

Elena Gagliardini, Biol SciD

Mario Negri Institute for Pharmacological Research, Negri Bergamo Laboratories Via Gavazzeni 11

IT-24125 Bergamo (Italy)

Tel. +39035319888, Fax +39035319331, E-Mail gagliardini@marionegri.it 
ic syndromes identified nephrin, a transmembrane protein of the immunoglobulin superfamily, which is predominantly expressed in glomerular podocytes [3-5]. Mutations of the nephrin gene in severe congenital nephrotic syndrome of the Finnish type [4] resulted in the lack of nephrin within the glomerulus, massive proteinuria in utero and nephrotic syndrome at birth, supporting the role of nephrin in the maintenance of the normal glomerular filtration barrier. Changes in nephrin expression associated with altered glomerular permeability to proteins were recently shown in acquired proteinuric renal diseases in experimental animals [6-8]. Studies investigating nephrin expression in non-congenital human glomerular diseases yielded conflicting results instead. The issue appears so controversial that some authors reported different findings in different studies. Furness et al. [9] showed that glomerular nephrin mRNA evaluated by PCR was significantly decreased in patients with minimal change nephrotic syndrome and in 1 case of membranous nephropathy. In contrast, expression of nephrin mRNA and protein was normal in pediatric patients with minimal change nephrosis, focal segmental glomerulosclerosis, or membranous nephropathy, while reduced in patients with IgA nephropathy [10]. Other authors have found an extensive reduction in immunofluorescence nephrin staining in glomeruli from adult patients with primary nephroses, regardless of the diagnosis [11]. These controversial results can be explained by the small number of patients studied, the lack of the simultaneous assessment of nephrin mRNA and protein expression, and the use of different antibodies directed against different domains of the nephrin molecule. The purpose of the present study was to systematically evaluate nephrin mRNA and protein expression in 28 renal biopsy samples from adult patients with glomerular disease and proteinuria and in normal controls. We used nonradioactive in situ hybridization and immunohistochemistry with antibodies directed against the extra- and intracellular nephrin. The recent observation that podocytes also express CD2-associated protein (CD2AP), an adapter molecule capable of binding the carboxy terminal domain of nephrin linking the slit diaphragm to the podocyte cytoskeleton [12], has contributed to shedding light on the slit diaphragm architecture [13]. To assess whether possible changes in nephrin distribution were shared by another podocyte protein, we also evaluated CD2AP by immunohistochemistry. Finally, we investigated the fine structure of the slit diaphragm by assessing the slit frequency, the presence of electron-dense slit diaphragms, and the width of the slit pores by electron microscopy.

\section{Patients and Methods}

This was a prospective, observational study that considered all consecutive patients admitted for a diagnostic kidney biopsy to the Renal Unit of the Azienda Ospedaliera of Bergamo from January 2001 to July 2002. The predefined inclusion criteria for all potentially eligible subjects were: (1) age > 18 years; (2) clinical indications for a kidney biopsy; (3) hypertension controlled by drugs such as diuretics, $\beta$-blockers and vasodilators; (4) no previous steroid or immunosuppressive therapy for at least 6 months; (5) no previous treatment with (or at least 2-month withdrawal from) angiotensin-converting enzyme (ACE) inhibitors or angiotensin II receptor antagonists that could potentially affect nephrin expression. This decision was made on the basis of available experimental studies showing normalization of nephrin expression after treatment with drugs that interfere with angiotensin II synthesis or biological activity [6, 14]; (6) no evidence of renovascular disease, obstructive uropathy, systemic disease or any other condition that could confound the study findings; (7) wellcontrolled blood pressure of $<140 / 90 \mathrm{~mm} \mathrm{Hg}$, and (8) informed consent.

As controls, specimens of normal kidney tissue from 5 patients undergoing nephrectomy for kidney adenocarcinoma were selected.

Table 1 summarizes the clinical features of the patients included in the study. In all patients, 24-hour urinary protein excretion and serum creatinine concentration were measured using an autoanalyzer (CX5; Beckman Instruments Inc., Fullerton, Calif., USA).

\section{Reagents}

Rabbit anti-human nephrin antiserum, raised against the extracellular domain of human nephrin (amino acid residues 668-923, Ig repeat domains 7 and 8), was obtained as previously described [6]. The specificity of the antiserum was checked in human nephrin transfected or untransfected HEK 293 cells. By Western blot analysis, the antibody was detected to bind a $185-$ to $200-\mathrm{kD}$ band obtained from a cell membrane preparation from transfected but not untransfected cells. Rabbit polyclonal antibody directed against the intracellular domain of mouse nephrin, obtained as previously described [15], was kindly provided by Dr. Lawrence B. Holzman. A rabbit polyclonal antibody raised against a recombinant protein corresponding to amino acids 350-639 mapping at the carboxy terminus of human CD2AP was purchased from Santa Cruz Biotechnology (Santa Cruz, Calif., USA).

Mouse nephrin antisense and sense riboprobes were prepared and labelled by in vitro transcription using digoxigenin uridine triphosphate (Roche, Milan, Italy) according to the manufacturer's protocol.

\section{Preparation of Nephrin Riboprobe and Nonisotopic in situ}

Hybridization

A 346-bp fragment of mouse nephrin cDNA (positions 11551501) coding for a portion of the extracellular domain was constructed by PCR amplification of the 2,400-bp fragment of mouse nephrin cDNA previously obtained [6], using the following primers: forward 5'-GGCGGAGCTCTGAGACAGTC, and reverse 5'-TCACGGTACCGCCCAGCTGC. The PCR product was gel purified and digested by restriction enzymes $S a c \mathrm{I}$ and $K p n \mathrm{I}$ and subcloned into $S a c I$ and $K p n I$ sites of pBluescript SK (Stratagene, Florence, Italy) between $\mathrm{T} 3$ and $\mathrm{T} 7$ promoters. The sequence of the nephrin riboprobe shows $82 \%$ homology to the human sequence. 
Table 1. Clinical parameters at the time of biopsy

\begin{tabular}{llrllll}
\hline Diagnosis & $\begin{array}{l}\text { Age } \\
\text { years }\end{array}$ & $\begin{array}{l}\text { Sex } \\
\text { M/F }\end{array}$ & $\begin{array}{l}\text { U-Prot } \\
\text { g/day }\end{array}$ & $\begin{array}{l}\text { S-Creat } \\
\mathrm{mg} / \mathrm{dl}\end{array}$ & \multicolumn{2}{c}{ Blood pressure, mm Hg } \\
\cline { 6 - 7 } & & & & & & \\
systolic & diastolic \\
MCN & $56 \pm 9$ & $4 / 3$ & $6.4 \pm 2.2$ & $2.1 \pm 0.5$ & $123 \pm 4$ & $79 \pm 3$ \\
FSGS & $57 \pm 6$ & $3 / 4$ & $6.6 \pm 2.3$ & $1.2 \pm 0.1$ & $124 \pm 3$ & $77 \pm 2$ \\
IgA & $41 \pm 4$ & $10 / 4$ & $2 \pm 0.4$ & $1.5 \pm 0.2$ & $128 \pm 4$ & $83 \pm 3$ \\
\hline
\end{tabular}

U-Prot = Urinary protein; S-Creat = serum creatinine; $\mathrm{MCN}=$ minimal change nephrosis; FSGS = focal segmental glomerulosclerosis; IgA = IgA nephropathy.

In situ hybridization for nephrin was performed as previously described [6] in a set of biopsies comprising samples from each patient group, and processed and developed simultaneously. Briefly, sections of Dubosq-Brazil-fixed, paraffin-embedded renal tissue were heat-fixed $\left(65^{\circ} \mathrm{C}, 30 \mathrm{~min}\right)$ and deparaffinized. After permeabilization with proteinase K (40 $\mu \mathrm{g} / \mathrm{ml}$, Sigma-Aldrich, Milan, Italy), sections were hybridized with the RNA probes at a final concentration of $0.4 \mathrm{ng} / \mu \mathrm{l}$ in $2 \times \mathrm{SSC}, 10 \%$ dextran sulfate, $1 \times$ Denhardt's solution, $20 \mathrm{~m} M$ Vanadyl Ribonucleoside Complex (Invitrogen, Life Technologies, San Diego, Calif., USA), 0.1 $M$ sodium phosphate, and incubated overnight in a moist chamber at $45^{\circ} \mathrm{C}$. After being washed in $0.2 \times \mathrm{SSC}$ and blocked with a buffer-blocking solution $(50 \mathrm{mg} / \mathrm{ml}$ dried skimmed milk, $150 \mathrm{mM} \mathrm{NaCl}$ in $100 \mathrm{~m} M$ Tris $\mathrm{HCl}, \mathrm{pH}$ 7.8) at room temperature for $30 \mathrm{~min}$, the sections were incubated with antidigoxigenin antibody conjugated with alkaline phosphatase (Roche) at a dilution of $1: 1,000$ for $45 \mathrm{~min}$ at $37^{\circ} \mathrm{C}$. Colorimetric detection with nitro blue tetrazolium salt and 5-bromo-4-chloro-3-indolyl phosphate (Roche) was then performed, and the sections were mounted in $60 \%$ glycerol and examined by light microscopy. The negative control included a hybridization step with the sense probe. Evaluation of nephrin expression was done by an investigator blinded to the study.

\section{Immunohistochemical Analysis}

Dubosq-Brazil-fixed, paraffin-embedded kidney sections were deparaffinized and rehydrated. For immunoperoxidase analysis of the nephrin extracellular domain, the sections were incubated for 30 min with $0.3 \% \mathrm{H}_{2} \mathrm{O}_{2}$ in methanol to quench endogenous peroxidase. Tissues were permeabilized in $0.1 \%$ Triton X-100 in PBS $0.01 M(\mathrm{pH} 7.2)$ for $30 \mathrm{~min}$ and sites of nonspecific binding were blocked by 30-min incubation with normal goat serum (Vector Laboratories, Burlingame, Calif., USA). All the above steps were carried out at room temperature. For evaluation of the nephrin intracellular domain and CD2AP, kidney samples were treated with proteinase- $\mathrm{K}$ $\left(20 \mu \mathrm{g} / \mathrm{ml}\right.$; Sigma-Aldrich) for $10 \mathrm{~min}$ at $37^{\circ} \mathrm{C}$, instead of Triton $\mathrm{X}-100$, followed by microwave and citrate buffer $(10 \mathrm{~m} M, \mathrm{pH} 6)$ incubations. Primary antibodies were diluted in PBS $/ 0.02 \%$ sodium azide and added overnight at $4{ }^{\circ} \mathrm{C}$ (anti-extracellular domain antibody pre-absorbed with normal human serum, diluted 1:200; antiintracellular domain antibody, 1:300; anti-CD2AP antibody, 1:100). Subsequent steps included incubations with the secondary antibody (biotinylated goat anti-rabbit IgG, Vector Laboratories), avidin-biotin peroxidase complex solution, and finally the development with diaminobenzidine. The sections were then counterstained with Harris hematoxylin (Biooptica, Milan, Italy). A set of biopsies compris- ing samples from each patient group was processed and developed simultaneously. Negative controls were obtained by omitting the primary antibody on a second section present on all the slides. Evaluation and scoring of nephrin and CD2AP expression was done by 1 investigator who was blinded to the biopsy groups. Seven glomeruli for each sample were analyzed and the signal intensity was graded on a scale of $0-3(0=$ no staining; $1=$ weak staining; 2 = staining of moderate intensity; 3 = strong staining).

\section{Electron Microscopy}

Small kidney tissue fragments from patients with minimal change disease, IgA nephropathy and controls ( $\mathrm{n}=3$ for each group) were fixed with $2.5 \%$ glutaraldehyde in $0.1 M$ cacodylate buffer ( $\mathrm{pH} 7.4)$ for $4 \mathrm{~h}$ at $4{ }^{\circ} \mathrm{C}$, washed in cacodylate buffer, and then post-fixed with a solution of tannic acid-glutaraldehyde (1\% tannic acid and $1 \%$ glutaraldehyde in $0.1 \mathrm{M}$ phosphate buffer, $\mathrm{pH} 7.4$ ) for $2 \mathrm{~h}$. The tannic acid fixative contributes to increasing the contrast and definition of extracellular structures in the glomerulus and reveals the isoporous substructure of the slit diaphragm [16]. The kidney fragments were then post-fixed in $1 \%$ osmium tetroxide for $1 \mathrm{~h}$ and after 1 additional wash in cacodylate buffer, they were dehydrated through ascending grades of alcohol and embedded in Epon resin. Semi-thin sections were stained with toluidine blue in borax and examined by light microscopy, in order to randomly select the center-most glomerulus. Ultra-thin sections $(60-100 \mathrm{~nm})$ were cut on an ultramicrotome (LKB Instruments, Milan, Italy), collected on copper grids, and stained with uranyl acetate and lead citrate [16]. Ultrastructural evaluations were done by electron microscopy (Morgagni 268D, Philips, Brno, Czech Republic).

\section{Morphometrical Analysis}

An average of 39 images of three glomeruli per patient was digitized (final magnification $\times 56,000$ ) and processed with the imageprocessing software (NIH Image v.1.6.). Glomerular basement membrane (GBM) length and slit diaphragm width (SDW) were calculated using a computer-assisted morphometric unit. For each image, the GBM profile and SDW were manually outlined using a line automatically measured in screen pixels. Exact enlargement was used to calculate the actual values of measured length using digitized images on a calibration grid (Ernest F. Fullam, Inc., Latham, N.Y., USA). The width was calculated by the harmonic mean of 100 slit pores (in nanometers) as follows:

$$
\mathrm{W}_{\mathrm{h}}=(8 / 3 \Pi) \times\left[\left(\mathrm{N} / \Sigma\left(1 / \mathrm{W}_{\mathrm{i}}\right)\right]\right.
$$



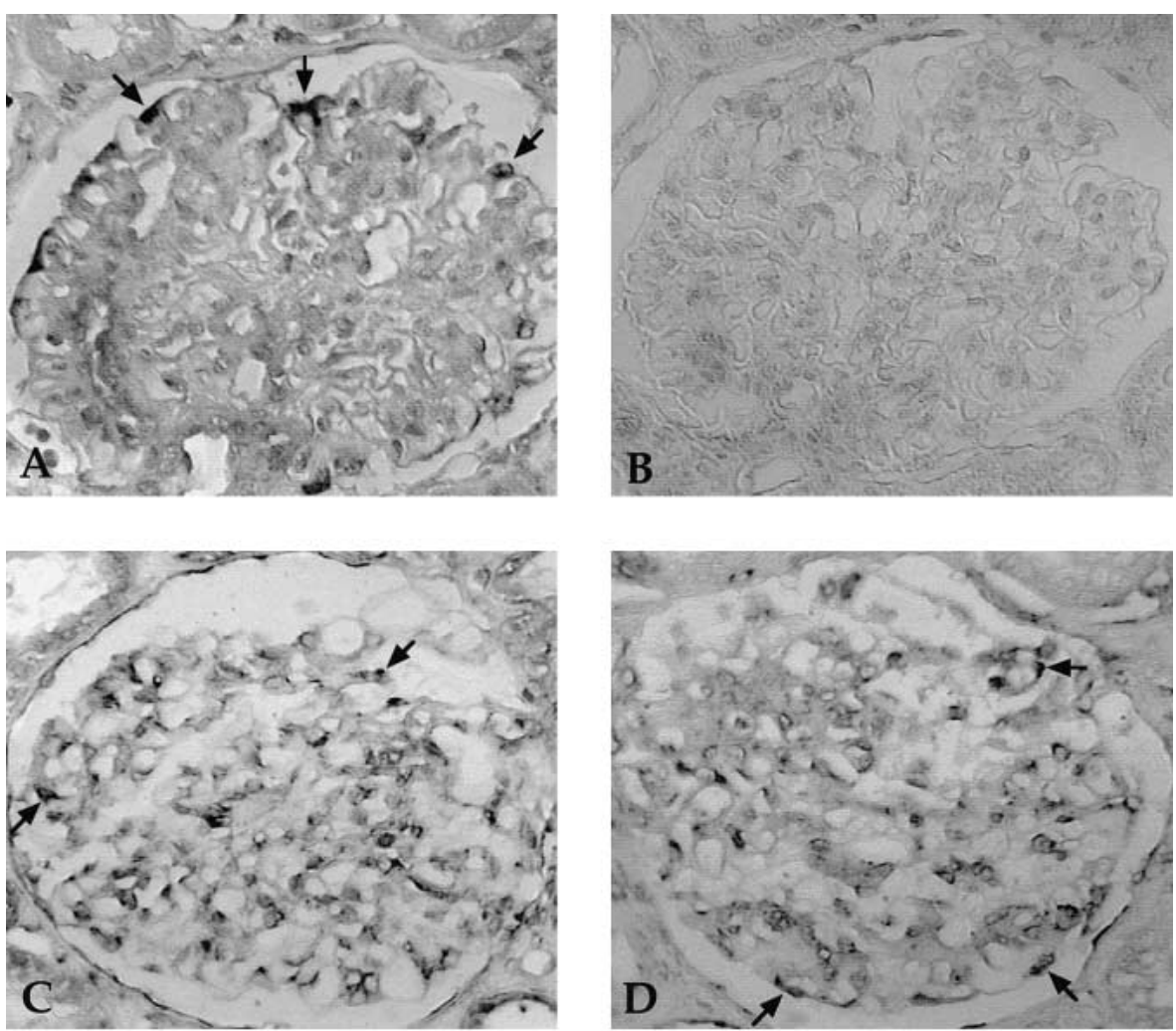

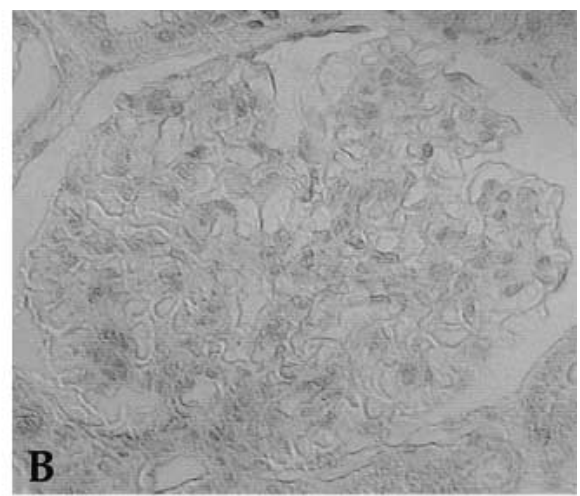

Fig. 1. Representative pictures of nephrin mRNA expression by in situ hybridization. Arrows indicate podocyte nephrin expression in control patients $(\mathbf{A})$ and in patients with minimal change nephrosis (C), focal segmental glomerulosclerosis (D) and IgA nephropathy (E). No specific signals were obtained with the sense nephrin probe (B). Original magnification, $\times 250$.

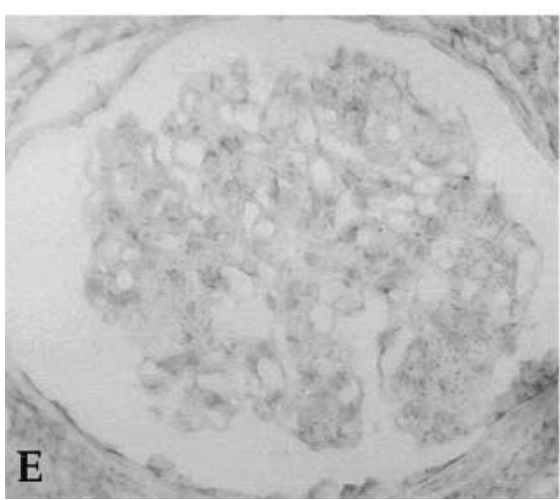

where $\mathrm{N}$ is the number of measures and $\mathrm{W}_{\mathrm{i}}$ is the measured width of the slit pore.

Epithelial filtration slit frequency was evaluated as the number of slits observed per micrometer of GBM length. The presence of the filamentous structure in the slit was evaluated in an average of 100 slits for each patient and expressed as percentage.

\section{Statistical Analysis}

Results are reported as the mean \pm SE. Differences between groups were analyzed by Kruskal Wallis test. A p value of $<0.05$ was considered significant.

\section{Results}

\section{Patients}

The study included 28 proteinuric patients and 5 nonproteinuric controls without histological evidence of glomerular disease. The histological diagnosis of proteinuric patients was minimal change disease in 7 cases, focal segmental glomerulosclerosis in 7 cases and IgA nephropathy in 14 cases. Table 1 summarizes the clinical features of the patients included in the study. In all patients, 24-hour 

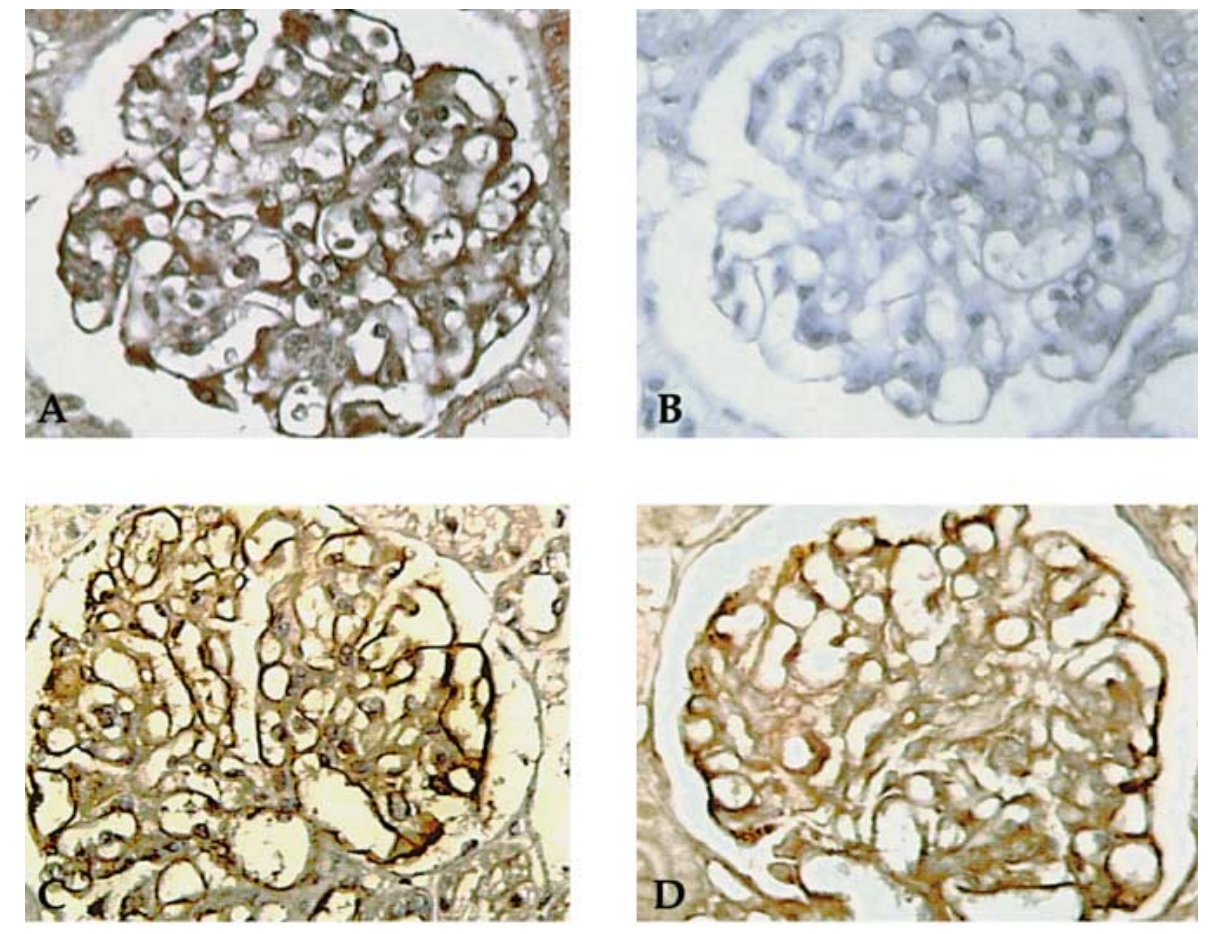

Fig. 2. Representative photomicrographs of the nephrin extracellular domain by the immunoperoxidase method in control patients (A) and in patients with minimal change nephrosis (C), focal segmental glomerulosclerosis (D) and IgA nephropathy (E). No specific signals were obtained omitting the primary antibody (B). Original magnification, $\times 250$.

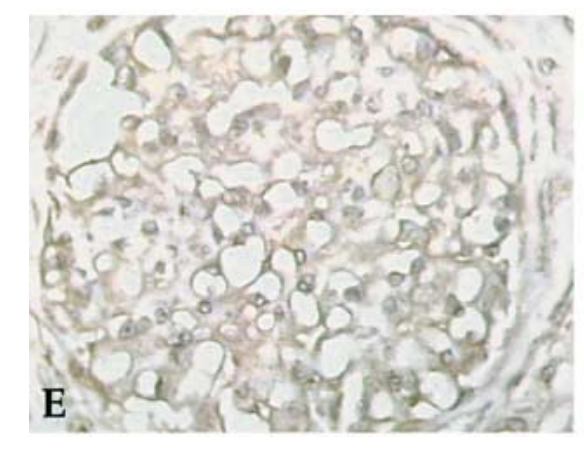

urinary protein excretion and the serum creatinine concentration were measured using an autoanalyzer.

\section{In situ Hybridization for Nephrin $m R N A$}

Nephrin mRNA expression was qualitatively evaluated in renal biopsies from patients with glomerular diseases and proteinuria and compared to control patients. While nephrin mRNA was comparable in glomeruli from patients with minimal change nephrosis (fig. 1C), focal segmental glomerulosclerosis (fig. 1D) and in control patients (fig. 1A), the intensity of the signal was markedly reduced in patients with IgA nephropathy (fig. 1E). Nephrin expression was mainly localized to the peripheral capillary loops of the glomerular tuft. Mild staining was occasionally observed in a few parietal epithelial cells and tubules resembling what we already observed in our previous study in rats with severe nephrosis [6]. No background signal was observed when the nephrin sense riboprobe was used indicating staining specificity (fig. 1B).

\section{Immunoperoxidase Staining for Nephrin}

Consistent with patterns of nephrin mRNA expression, immunohistochemistry in control patients with the antibody against the extracellular nephrin portion showed a prominent localization along the peripheral capillary loops (fig. 2A; score $2 \pm 0.3$ ). Nephrin staining comparable to that of controls was observed in the glomeruli of patients with minimal change nephrosis (fig. 2C; score $1.23 \pm 0.5)$ and focal segmental glomerulosclerosis (fig. $2 \mathrm{D}$; score $1.8 \pm 0.5$ ). The staining intensity varied 

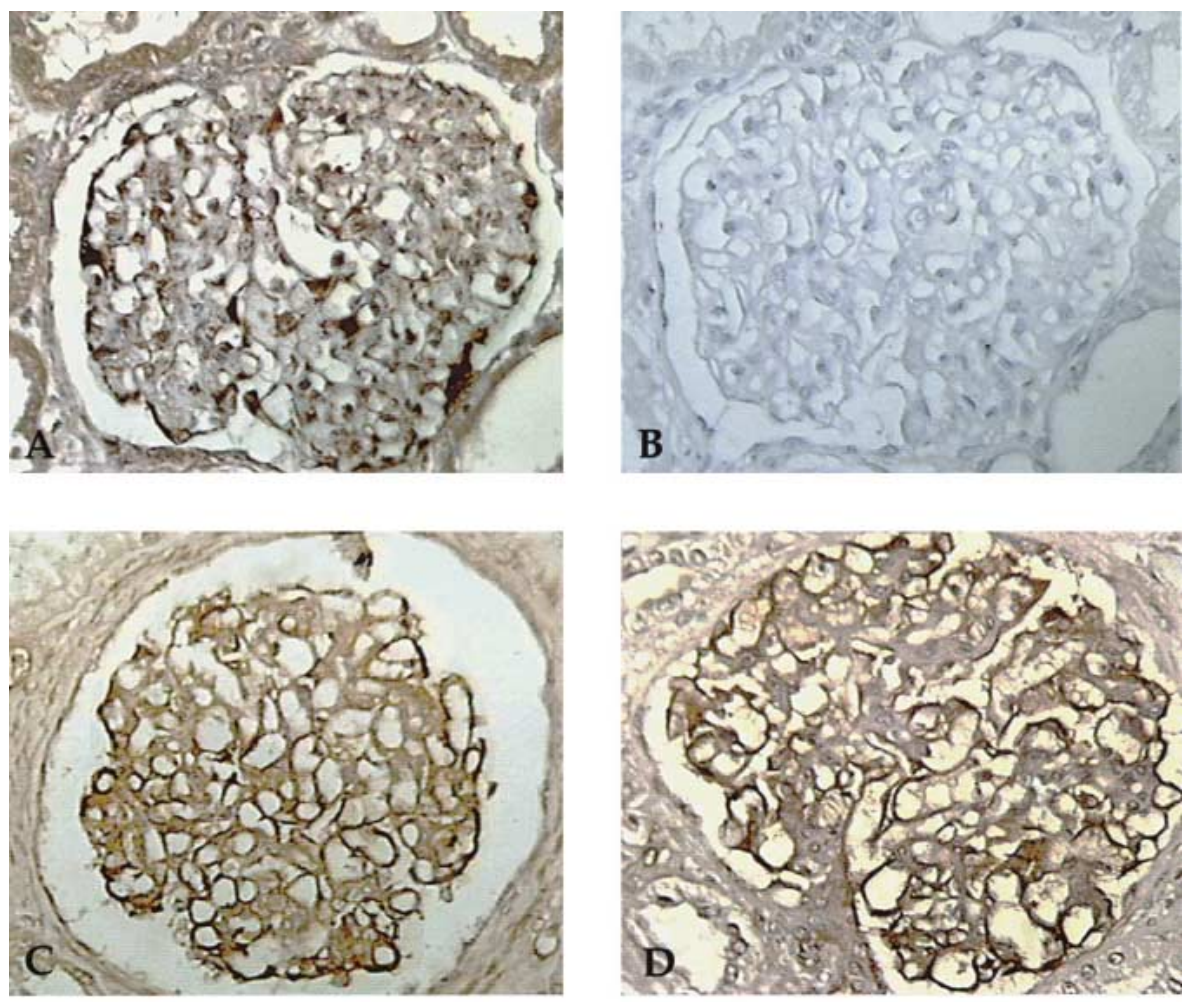

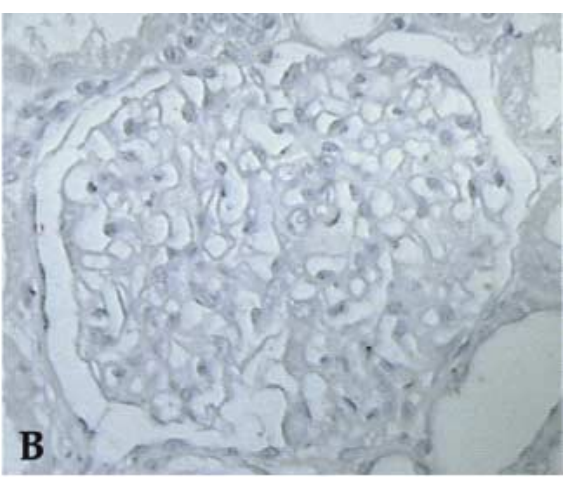

Fig. 3. Immunoperoxidase staining for nephrin intracellular domain in control patients (A) and in patients with minimal change nephrosis (C), focal segmental glomerulosclerosis (D) and IgA nephropathy (E). No specific signals were obtained omitting the primary antibody (B). Original magnification, $\times 250$.

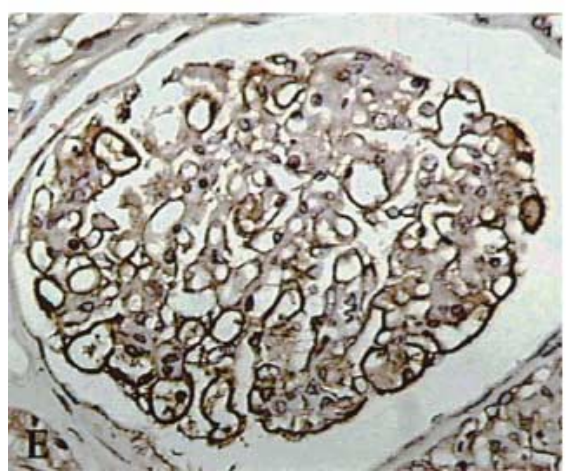

somewhat along the capillary wall and in podocytes. In particular, sclerotic areas were negative (data not shown). Tubules were occasionally stained. As shown in figure 2, the staining intensity was significantly reduced in patients with IgA nephropathy (fig. 2E; score $0.4 \pm 0.1$ ) compared to control patients $(\mathrm{p}<0.05)$. Nephrin staining was completely abrogated omitting the primary antibody indicating staining specificity (fig. 2B).

More homogeneous results were observed when the antibody specific for intracellular domain of nephrin was used. In normal glomeruli, nephrin exhibited an epithelial pattern with a linear distribution along the peripheral capillary loops (fig. 3A). Independent of the disease, all pa- tients maintained a nephrin staining intensity comparable to that observed in control patients, with a peripheral positivity absent only in sclerotic areas (fig. 3C-E). No specific signal omitting the primary antibody was observed (fig. 3B).

\section{Immunoperoxidase for $C D 2 A P$}

Since CD2AP seems to play a key role in anchoring nephrin to the podocytes, we evaluated whether CD2AP expression changed in the glomerular disease patients considered here (fig. 4). In normal glomeruli, CD2AP showed a prominent glomerular localization. A linear dotted line of reactivity could be observed giving a preferen- 

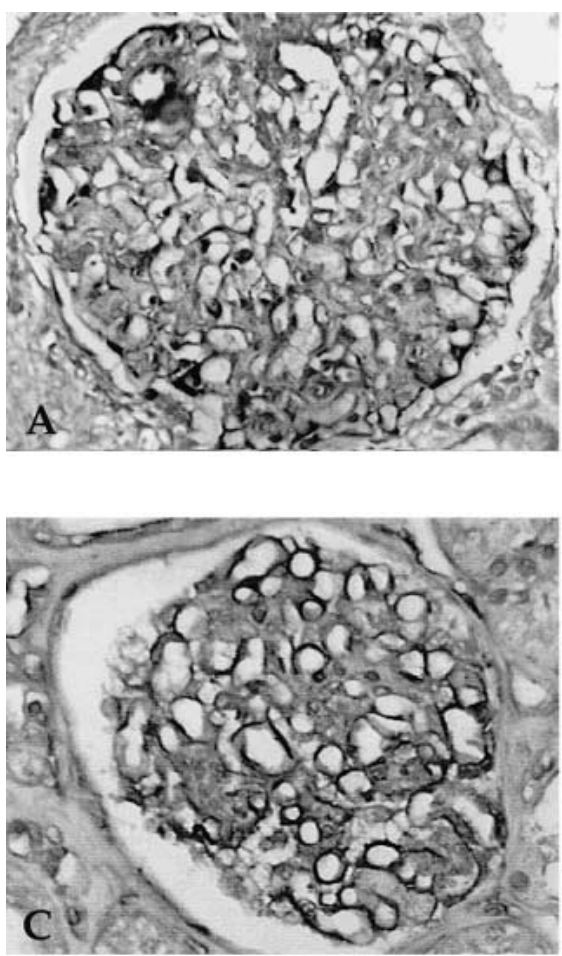
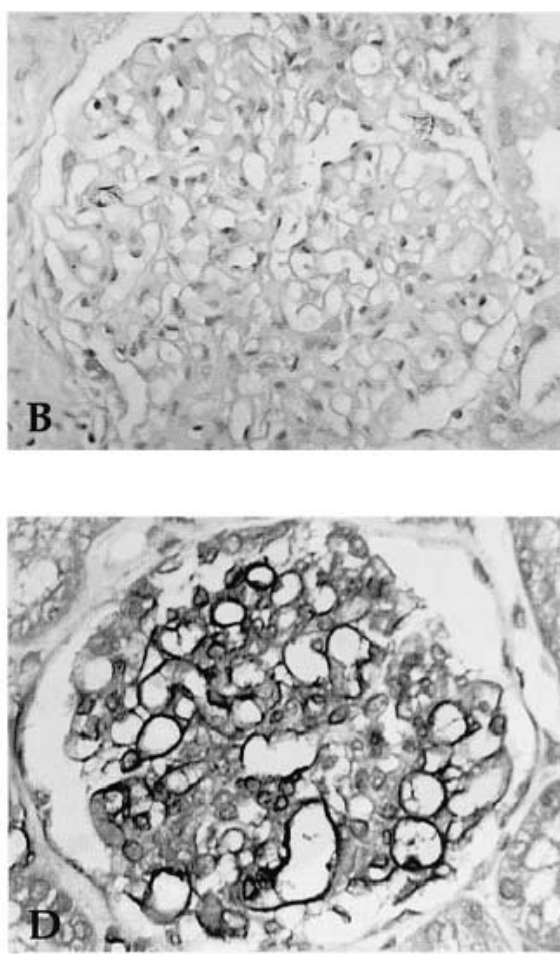

Fig. 4. Immunoperoxidase staining for CD2AP in control patients (A) and in patients with minimal change nephrosis $(\mathbf{C})$, focal segmental glomerulosclerosis (D) and IgA nephropathy (E). No specific signals were obtained omitting the primary antibody (B). Original magnification, $\times 250$.

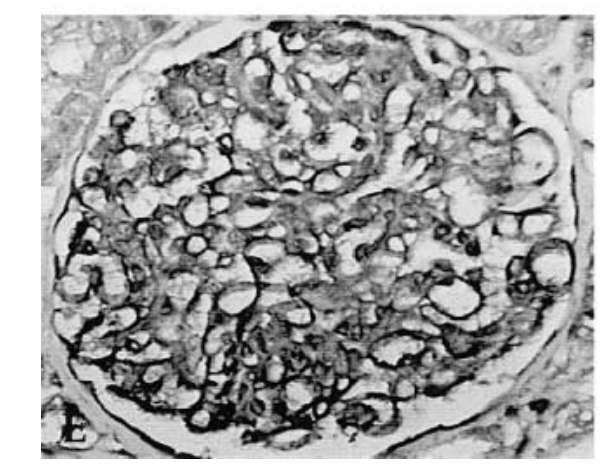

tially epithelial-like staining pattern (fig. 4A). No difference was seen between the patients who maintained a strong positivity along the peripheral capillary loops (fig. 4C-E). No specific signal was observed by omitting the primary antibody (fig. 4B). The appreciable difference between nephrin and CD2AP staining suggests that changes in nephrin protein were specific and not part of a generalized phenomenon.

\section{Electron Microscopy Studies}

We further investigated the integrity of the filtration slit by electron microscopy taking advantage of kidney tissue post-fixation with tannic acid and glutaraldehyde. This method lends considerable contrast and definition to the extracellular structures due to tannic acid's ability of forming extremely strong complexes with proteins. The frequency of the slit pores along the GBM was remarkably reduced in patients with minimal change disease in comparison to controls $(0.98 \pm 0.04$ vs. $2.2 \pm 0.2 \mathrm{slits} / \mu \mathrm{m}$ GBM) due to extensive foot process effacement. In IgA nephropathy the frequency of the slit pores did not change $(1.96 \pm 0.06 \mathrm{slits} / \mu \mathrm{m}$ GBM$)$. The percentage of slit diaphragms with a filamentous image was comparable in controls and patients with minimal change disease, averaging 62 and $60 \%$, respectively. This percentage compares reasonably well with that of the available studies in the literature that have addressed this issue $[4,17,18]$. In contrast, a dramatic reduction in the percentage of elec- 

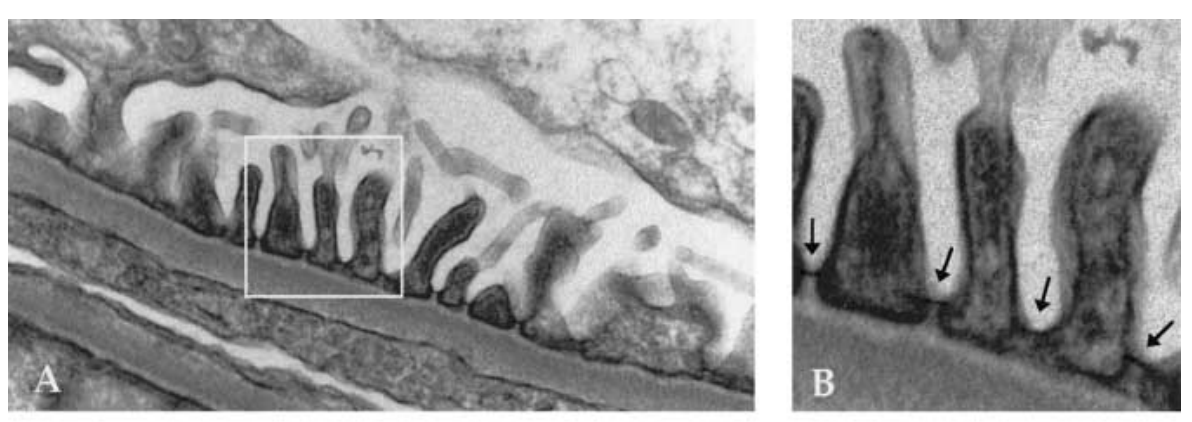

Fig. 5. Representative transmission electron micrographs of the ultrastructure capillary wall in control patients $(\mathbf{A}, \mathbf{B})$ and in patients with IgA nephropathy (C, D). B, D The enlarged details of A and C. Arrows indicate electron-dense diaphragms in the filtration slits. Original magnification, $\times 22,000$.
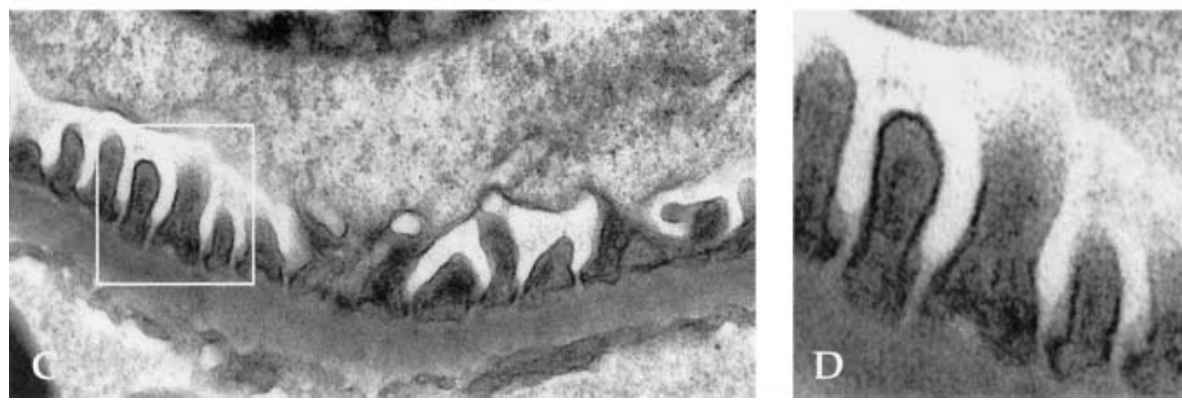

tron-dense slit diaphragms was observed in the patients with IgA nephropathy (17\%). The width of the slit pore calculated as a harmonic mean showed a tendency to decrease in patients with minimal change disease (20.01 $\pm 0.5 \mathrm{~nm})$ and $\operatorname{IgA}$ nephropathy $(21.85 \pm 0.4 \mathrm{~nm})$ when compared to control subjects $(25 \pm 2.9 \mathrm{~nm})$. Representative pictures of the ultrastructure of the capillary wall are shown in figure 5.

\section{Discussion}

Here for the first time, nephrin mRNA expression was assessed systematically in all patients recruited for the study in association with immunostaining of the corresponding protein which was done in each patient using antibodies with separate specificities, one recognizing the extracellular domain and the other the intracellular portion of the molecule. Glomerular expression of nephrin mRNA and protein was not different in patients with minimal change nephrosis and focal segmental glomerulosclerosis in comparison to control patients. In contrast, a remarkable reduction or even absence of nephrin mRNA and staining intensity for the extracellular nephrin domain was found in IgA nephropathy patients in the face of a normal expression of the intracellular nephrin domain.

The extracellular portion of the nephrin molecule contains eight Ig motifs characteristic of proteins participat- ing in cell-cell interaction and one fibronectin type-IIIlike module. The intracellular domain has nine tyrosine residues characteristic of signaling adhesion molecules [19]. The location of nephrin in the slit diaphragm as documented by previous immunoelectron microscopy studies [20] together with the fact that nephrin molecules from two adjacent podocytes extend towards each other and behave as adhesion proteins led to hypothesize on the homophilic interactions of the two nephrin molecules at the podocyte slit diaphragm [19]. Early evidence concurs to indicate that nephrin is essential for the functional integrity of the glomerular filter. Besides human congenital nephroses characterized by both the lack of nephrin in the slit diaphragm and heavy proteinuria, injection into rats of the nephritogenic monoclonal 5-1-6 antibody against the extracellular domain of nephrin [21] caused pronounced proteinuria and redistribution of nephrin from a linear to a granular pattern without induction of structural changes in the filtration slit [22]. In a severe model of passive Heymann nephritis reminiscent of human membranous nephropathy, the glomerular expression of nephrin mRNA and protein decreased over time in parallel to the development of proteinuria and the subsequent renal damage [6].

The present observation of normal nephrin expression in minimal change disease seems in keeping with recent electron microscopy findings that, in patients with minimal change disease, nephrin is normally expressed in the 
podocytes displaying intact foot processes [23]. The findings above showing that nephrin is no longer detectable at sites of podocyte effacement likely reflect a major rearrangement of the cell cytoskeleton structure which may conceivably impair the binding of the antibody to the nephrin epitope. Here we also found a comparable percentage of slit diaphragms with the filamentous image in controls and minimal change disease patients, while the percentage of slit diaphragms with a filamentous image was reduced in IgA nephropathy. Collectively, these findings are consistent with nephrin gene and protein expression data indicating major abnormalities confined to IgA. Our results are also in agreement with findings in pediatric and adult kidney disease patients $[10,24]$ and very recent data from Wang et al. [25], but are at variance with those of Doublier et al. [11] who, however, may have selected patients in a very early phase of the disease, at least for IgA nephropathy, to the extent that in their group urinary protein excretion was normal.

Nephrin is linked to CD2AP, a molecule that bridges the cytoplasmic domain of nephrin to the podocyte cytoskeleton, both contributing to slit diaphragm integrity. Mice lacking CD2AP exhibit a nephrotic syndrome that resembles the congenital form of nephrosis due to nephrin mutations and die of renal failure within 2 months of birth [13]. The absence of CD2AP in knockout mice interferes with the formation of the interdigitating foot processes and the slit diaphragm and with expression of nephrin in foot processes [13]. We assessed whether the abnormalities of nephrin could be shared by CD2AP. Comparable CD2AP expression was found in all acquired nephroses examined here compared to control patients, suggesting that changes in IgA nephropathy patients are confined to nephrin.

To reconcile the results presented here of lower than normal nephrin gene expression and corresponding protein staining in IgA nephropathy but not in minimal change nephrosis and focal segmental glomerulosclerosis, one may possibly consider that the glomerular lesions of IgA nephropathy ensue with the expansion of the mesangial matrix. Mesangial expansion induces alterations of the glomerular tuft leading to phenotypic changes in podocytes [26] that may eventually result in remodelling and rearrangement of nephrin molecules in the slit diaphragm. Another possible explanation for the selective reduction in extracellular nephrin rests on the possibility that different spliced variants of nephrin mRNA could be expressed in IgA nephropathy as previously suggested in experimental animals. Thus, a soluble form of nephrin was recently found in urine from rats with diabetes or glo- merular disease by means of immunoblotting with an antibody that recognizes the extracellular part of the molecule $[8,27]$. This phenomenon was attributed to the excretion of an alternative spliced form of nephrin [8]. Finally, it is interesting to note that IgA nephropathy responds to ACE inhibition in terms of a reduction in proteinuria which is at variance to that observed in minimal change and focal segmental glomerulosclerosis [28]. Experimental studies suggest that drugs that block angiotensin II synthesis/biological activity effectively reduced proteinuria and fully prevented the decrease in nephrin expression [6,29]. Along this line, studies aimed at evaluating nephrin mRNA and protein expression in renal biopsies before and after ACE inhibitor treatment should be relevant.

In the present study the use of two different antibodies allowed us to appreciate that extracellular nephrin is extremely reduced, while intracellular nephrin is normally expressed. With this approach we could carefully assess the abnormalities of the nephrin molecule which are at variance with previous studies that used a single antibody against a single portion of the protein giving only a partial representation of glomerular nephrin expression.

In summary, nephrin mRNA and expression of extracellular nephrin were found to be selectively reduced in patients with IgA nephropathy, in the presence of normal staining intensity of the intracellular part of the molecule. Finding that nephrin expression is normal in minimal change and focal segmental glomerulosclerosis suggests that the reduction observed in IgA is not a consequence of proteinuria. Downregulation of nephrin is a specific marker of the disease to the extent that another slit diaphragm protein CD2AP was normal. Nephrin changes in IgA nephropathy parallel a reduction in the percentage of slit diaphragms in which a filamentous structure could be observed by electron microscopy. Such changes are likely to account for proteinuria in IgA nephropathy.

\section{Acknowledgments}

The authors wish to express their gratitude to Prof. Antonino Lembo and Marlisa Capitanio for their invaluable collaborative effort in obtaining specimens from patients undergone nephrectomy. The authors are also deeply indebted to Dr. Lawrence Holzman for kindly providing the antibody recognizing the intracellular portions of nephrin. We thank Dr. Andrea Remuzzi for help with the morphometric evaluation of the slit diaphragm on electron microscopy.

E.G. is the recipient of a fellowship in memory of Dr. Luigi and Tilde Bianchi from the 'Associazione Ricerca Malattie Rare', Bergamo, Italy. The study was supported in part by a grant from the Associazione Trenta Ore per la Vita (Rome, Italy). 


\section{References}

1 Falk RJ, Jennette JC, Nachman PH: Primary glomerular disease; in Brenner BM (ed): The Kidney. Philadelphia, Saunders, 2000, pp 1263-1349.

2 Appel GB, Radhakrishnan J, D'Agati D: Secondary glomerular disease; in Brenner BM (ed): The Kidney. Philadelphia, Saunders, 2000, pp 1350-1448.

3 Kestilä M, Lenkkeri U, Männikkö M, et al: Positionally cloned gene for a novel glomerular protein - nephrin - is mutated in congenital nephrotic syndrome. Mol Cell 1998;1:575582.

4 Patrakka J, Kestila M, Wartiovaara J, et al: Congenital nephrotic syndrome (NPHS1): Features resulting from different mutations in Finnish patients. Kidney Int 2000;58:972-980.

5 Lenkkeri U, Mannikko M, McCready P, et al: Structure of the gene for congenital nephrotic syndrome of the Finnish type (NPHS1) and characterization of mutations. Am J Hum Genet 1999;64:51-61.

6 Benigni A, Tomasoni S, Gagliardini E, et al: Blocking angiotensin II synthesis/activity preserves glomerular nephrin in rats with severe nephrosis. J Am Soc Nephrol 2001;12:941948.

7 Luimula P, Ahola H, Wang SX, et al: Nephrin in experimental glomerular disease. Kidney Int 2000;58:1461-1468.

8 Aaltonen P, Luimula P, Astrom E, et al: Changes in the expression of nephrin gene and protein in experimental diabetic nephropathy. Lab Invest 2001;8:1185-1190.

9 Furness PN, Hall LL, Shaw JA, et al: Glomerular expression of nephrin is decreased in acquired human nephrotic syndrome. Nephrol Dial Transplant 1999;14:1234-1237.

10 Patrakka J, Ruotsalainen V, Ketola I, et al: Expression of nephrin in pediatric kidney diseases. J Am Soc Nephrol 2001;12:289-296.
11 Doublier S, Ruotsalainen V, Salvidio G, et al: Nephrin redistribution on podocytes is a potential mechanism for proteinuria in patients with primary acquired nephrotic syndrome. Am J Pathol 2001;158:1723-1731.

12 Li C, Ruotsalainen V, Tryggvason $\mathrm{K}$, et al: CD2AP is expressed with nephrin in developing podocytes and is found widely in mature kidney and elsewhere. Am J Physiol Renal Physiol 2000;279:F785-F792.

13 Shih NY, Li J, Karpitskii V, et al: Congenital nephrotic syndrome in mice lacking CD2-associated protein, Science 2001;286:312-315.

14 Bonnet F, Cooper ME, Kawachi H, et al: Irbesartan normalises the deficiency in glomerular nephrin expression in a model of diabetes and hypertension. Diabetologia 2001;44:874-877.

15 Holzman LB, St John PL, Kovari IA, et al: Nephrin localizes to the slit pore of the glomerular epithelial cell. Kidney Int 1999;56:14811491.

16 Rodewald R, Karnovsky MJ: Porous substructure of the glomerular slit diaphragm in the rat and mouse. J Cell Biol 1974;60:423-433.

17 Ruotsalainen V, Patrakka J, Tissari P, et al: Role of nephrin in cell junction formation in human nephrogenesis. Am J Pathol 2001;157: 1905-1916.

18 Patrakka J, Ruotsalainen V, Reponen P, et al: Recurrence of nephrotic syndrome in kidney grafts of patients with congenital nephrotic syndrome of the Finnish type. Transplantation 2002;73:394-403.

19 Tryggvason K: Unraveling the mechanisms of glomerular ultrafiltration: Nephrin, a key component of the slit diaphragm. J Am Soc Nephrol 1999; 10:2440-2445.

20 Ruotsalainen V, Ljungberg P, Wartiovaara J, et al: Nephrin is specifically located at the slit diaphragm of glomerular podocytes. Proc Natl Acad Sci USA 1999;96:7962-7967.
21 Topham PS, Kawachi H, Haydar SA, et al: Nephritogenic mAb 5-1-6 is directed at the extracellular domain of rat nephrin. J Clin Invest 1999;104:1559-1566.

22 Orikasa M, Matsui K, Oite T, et al: Massive proteinuria induced in rats by a single intravenous injection of a monoclonal antibody. $\mathrm{J}$ Immunol 1988;141:807-814.

23 Huh W, Kim DJ, Kim M-K, et al: Expression of nephrin in acquired human glomerular disease. Nephrol Dial Transplant 2002;17:478484.

24 Verkade MA, Baelde JJ, Eikmans M, et al: Altered glomerular mRNA levels for nephrin and podoplanin in minimal change disease and focal and segmental glomerulosclerosis (abstract). J Am Soc Nephrol 2001;12:695A696A.

25 Wang S-X, Rastaldi MP, Patari A, et al: Patterns of nephrin and a new proteinuria-associated protein expression in human renal diseases. Kidney Int 2002;61:141-147.

26 Kretzler M, Koeppen-Hagemann I, Kriz W Podocyte damage is a critical step in the development of glomerulosclerosis in the uninephrectomised-desoxycorticosterone hypertensive rat. Virchows Arch 1994;425:181-193

27 Luimula P, Aaltonen P, Ahola H, et al: Alternatively spliced nephrin in experimental glomerular disease of the rat. Pediatr Res 2000;48. 759-762.

28 Ruggenenti P, Schieppati A, Remuzzi G: Progression, remission, regression of chronic renal diseases. Lancet 2001;357:1601-1608.

29 Cao Z, Bonnet F, Candido R, et al: Angiotensin type 2 receptor antagonism confers renal protection in a rat model of progressive renal injury. J Am Soc Nephrol 2002;13:1773-1787. 